\title{
Could humans recognize odor by phonon assisted tunneling?
}

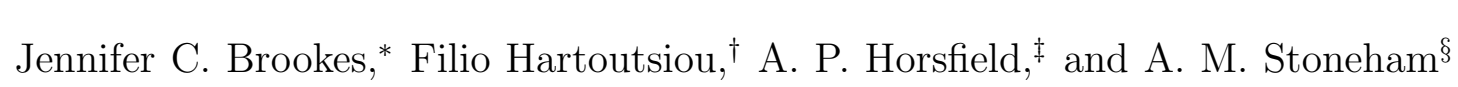 \\ Department of Physics and Astronomy, \\ University College London, Gower Street, \\ London WC1E 6BT, United Kingdom
}

\begin{abstract}
Our sense of smell relies on sensitive, selective atomic-scale processes that are initiated when a scent molecule meets specific receptors in the nose. However, the physical mechanisms of detection are not clear. While odorant shape and size are important, experiment indicates these are insufficient. One novel proposal suggests inelastic electron tunneling from a donor to an acceptor mediated by the odorant actuates a receptor, and provides critical discrimination. We test the physical viability of this mechanism using a simple but general model. Using values of key parameters in line with those for other biomolecular systems, we find the proposed mechanism is consistent both with the underlying physics and with observed features of smell, provided the receptor has certain general properties. This mechanism suggests a distinct paradigm for selective molecular interactions at receptors (the swipe card model): recognition and actuation involve size and shape, but also exploit other processes.
\end{abstract}

\footnotetext{
${ }^{*}$ Electronic address: j.brookes@ucl.ac.uk

${ }^{\dagger}$ Electronic address: to.milaraki@hotmail.com

${ }^{\ddagger}$ Electronic address: a.horsfield@ucl.ac.uk

${ }^{\S}$ Electronic address: a.stoneham@ucl.ac.uk
} 
Our sense of smell affects our behavior profoundly. Discrimination between small molecules, often in very low concentrations, allows us to make judgments about our immediate environment [1] and influence our perceptions. Even though odorants are key components of many commercial products[2], the biomolecular processes of olfaction are inadequately understood: scent design is not straightforward. We know that odor detection involves several types of receptor for a given odorant, and understand how a receptor signal is amplified and processed[3, 4]. However, the initial selective atomic-scale processes as the scent molecule meets its nasal receptors are not well understood. Odorant shape and size are certainly important, but experiment shows these are insufficient. Here we assess the novel proposal that a critical early step involves inelastic electron tunneling mediated by the odorant. We test the physical viability of this mechanism[5] using electron transfer (ET) theory, with values of key parameters in line with those for other biomolecular systems. The proposed mechanism is viable (there are no physics-based objections and is consistent with known features of olfaction) provided the receptor has certain general properties. This mechanism has wider importance because it introduces a distinct paradigm for selective actuation of receptors: whereas lock and key models[6] imply size, shape and non-bonding interactions (the docking criteria) are all, in our swipe card model recognition and actuation involve other processes in addition to docking. Thus it encompasses and goes beyond mechanisms such as proton transfer, discussed by us previously[7].

All current theories agree that selective docking of odorants is important[2]. However, odorants are small molecules (rarely more than a few tens of atoms[2]), and it is improbable that docking criteria alone offer sufficient discrimination. For example, molecules with almost identical shapes can smell very different: replacing carbon with its isosteres $\mathrm{Si}$, Ge and Sn invariably markedly alter odor character[8]. Something more is needed for olfaction, leading to early suggestions that odorant vibration frequencies were critical \9, 10》, though without specific mechanisms. Both infrared and inelastic electron tunneling [11, 12] (IETS) spectroscopies distinguish very precisely between different molecules through vibrational frequencies and intensities, which makes the proposal appealing. The first specific mechanism (based on IETS) was Turin's[13] idea that there is odorant mediated inelastic tunneling of an electron at the receptor: inelastic tunneling between receptor electronic states differing in energy by $\hbar \omega$ that occurs only when energy is conserved by emission of an odorant phonon of the right energy, hence selectivity. Clearly, the vibration must also couple to the electronic 
transition.

Experiment offers several tests of Turin's idea. It explains why certain molecules with very different shapes can smell similar (e.g. boranes and thiols), but also why some molecules of essentially identical shape smell utterly different (e.g. 1,1-dimethylcyclohexane and its sila counterpart) because of frequency or coupling changes. The question of whether humans can distinguish between a molecule and its deuterated counterpart is still controversial. There is evidence both for [13, 14] and against[15]. In animals, isotope discrimination is well-documented[16]. Both left- and right-handed forms of enantiomers should have the same vibrational spectrum. The odors of some enantiomer pairs are the same (type 1) while others differ (type 2) [17]. Type 2 can be explained by docking criteria (different chiralities fit different receptors), while type 1 is naturally explained by vibrational frequency. However, docking and frequency together can account for both since chirality will affect the intensity of response of receptors to the enantiomers (the helices that form the walls of the receptors are chiral). Quantitative support for the theory comes from the successful correlation of odor character with tunneling frequency spectrum[18] for a range of odorants. Indeed, vibrational frequency has been found to correlate better with odor than structure [19]. Thus a molecule's vibrational spectrum appears closely linked to its odor. We now test whether the physical processes underlying Turin's proposed mechanism for detecting the frequency are credible.

First we focus on the odorant and perform a simple test of whether odor can be related to vibrational frequency and to coupling to the odorant charges (as required by IETS). We computed the vibrational spectra of $\mathrm{H}_{2} \mathrm{~S}$ and four boranes (decaborane, m-, o- and pcarborane). The boranes are structurally similar, but all quite distinct from $H_{2} S$. However, $\mathrm{H}_{2} \mathrm{~S}$ and decaborane smell sulfuraceous, while the carboranes smell camphoraceous. Using Gaussian 03[20] we computed vibrational frequencies and infrared (IR) couplings, defined as $\left|\partial \vec{p} / \partial Q_{i}\right|^{2}$ with $\vec{p}$ the dipole moment and $Q_{i}$ a displacement along normal mode $i$. The sulfuraceous smell of $\mathrm{H}_{2} S$ is associated with vibrations in the region of $2600 \mathrm{~cm}^{-1}$ [21]. In this region decaborane has IR couplings that are one to two orders of magnitude greater than the carboranes. Assuming that the IR couplings are a good estimate of the electron-oscillator coupling in an olfactory receptor, this could explain sulfuraceous and less sulfuraceous odors.

Turning to the combined odorant and receptor system, we must determine whether ET is possible on the relevant time scale, and if so whether the discriminating electron transfer rate (with excitation of the critical odorant mode) is sufficiently large relative to rates 
for non-discriminatory transfer channels (without excitation of the critical mode). This is necessary because most IETS experiments observe inelastic tunneling with phonon emission as a weak adjunct to the elastic component, detected only by complex post-processing of a type unlikely in a nasal environment. Too little is currently known about the atomic and electronic structure of real odorant receptors for full-scale calculations. Instead we make general assumptions about the nature of the receptor, the odorant and their interaction. These assumptions relate to a series of characteristic times corresponding to the required physical processes (see Fig. 11).

Turin's theory requires a source of electrons or holes to allow charge flow to take place. The precise biological origin is not known, but may well consist of reducing (oxidizing) species $(X)$ in the cell fluid [2]. These molecules diffuse through the aqueous medium and arrive with an average interval of $\tau_{X}$. Using a standard approach for computing reactant collision rates in solution from the diffusion equation and the Stokes-Einstein relation for the diffusion coefficient [22] we get $\tau_{X}=3 \eta / 2 n_{X} k_{B} T$ where $\eta$ is the viscosity of water $(0.891 \times$ $\left.10^{-3} \mathrm{kgm}^{-1} \mathrm{~s}^{-1}\right), n_{X}$ is the concentration of $X, k_{B}$ is Boltzmann's constant and $T$ is the temperature. Note that this result is independent of the nature of $X$ or the receptor. Since $n_{X}$ will probably lie in the range $1 \mu \mathrm{M} \rightarrow 100 \mu \mathrm{M}$, we get a range of values for $\tau_{X}$ of $10 \mu \mathrm{s} \rightarrow 1 \mathrm{~ms}$. The charge now has to cross from the molecule to the receptor molecule, a process that can be described by Marcus theory [23, 24, 25], and characterized by a time $\tau_{I}$. In proteins times range from about $1 \mathrm{~ms}$ down to about $1 \mu \mathrm{s}[26]$. The injected charge has to propagate through to the donor $(D)$. The route is not known, but probably involves hopping transport. Thus the journey time is likely be in the ms to $\mu$ s range as for charge injection. The next step is the inelastic tunneling from $D$ to $A$ (the acceptor), and it is this charge movement that actuates the receptor. Now the charge must reach the mechanism that releases the G-protein which in turn initiates the signal that is sent to the brain. Again, we do not know the route taken but is likely to involve charge hopping. So the characteristic time $\tau_{R}$ will probably be in the range ms to $\mu$ s. Thus, overall charge injection and extraction together are likely to occur on typical biological time scales of $\mu$ s to ms.

For the mechanism to work there must be essentially no tunneling from $D$ to $A$ in the absence of the odorant, either because the distance is too great or energy conservation is problematic. The odorant must make inelastic transmission possible by a mechanism coupling electron movement from $D$ and $A$ to vibrational excitation in the odorant. In 


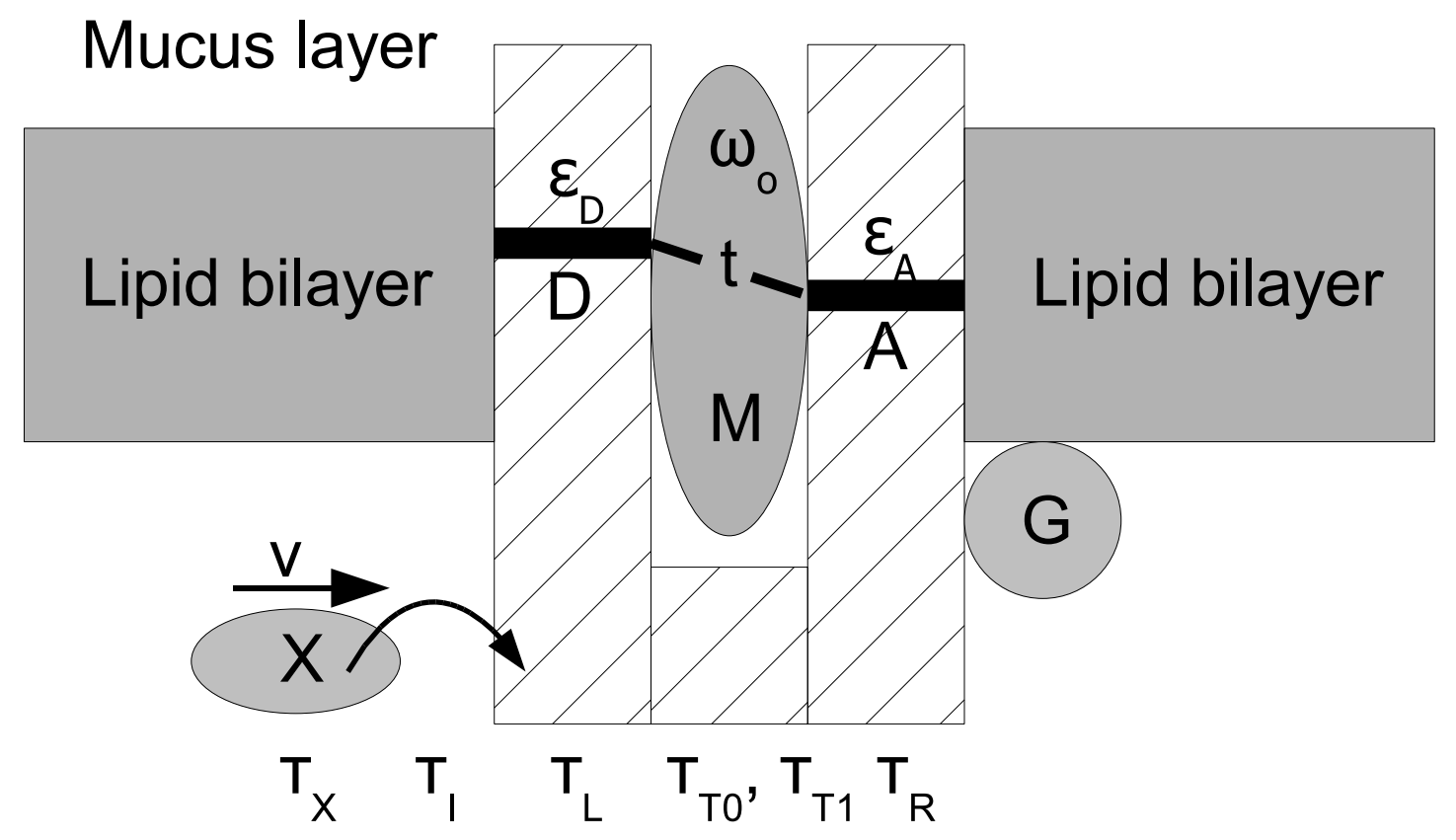

FIG. 1: The olfactory receptor is a G-protein coupled receptor with seven hydrophobic helices that span the cell membrane. It responds to the arrival of a recognized odorant by releasing the $\alpha$-subunit of a neighboring G-protein, which in turn initiates a large influx of Ca ions into the cell, a signal that can be communicated to the brain. This figure represents the model of the receptor we use to describe its action. The electron (hole) source $X$ is likely to be a reducing (oxidizing) agent in the cell fluid. It arrives at a site on the outside of the receptor protein where it can exchange charge after an average interval $\tau_{X}$. Once in place, it exchanges a charge with a characteristic time $\tau_{I}$. The charge then travels to the donor $D$ in one transmembrane helix of the protein over an average time of $\tau_{L}$, from where it then hops to the acceptor $A$ (possibly in a different helix) with either an average time $\tau_{T 0}$ for non-discriminating ("elastic") tunneling or an average time $\tau_{T 1}$ for discriminating ("inelastic") tunneling. Only the inelastic contribution is sensitive to the odorant $(M)$ oscillator frequency $\omega_{0}$, and so needs to dominate the elastic contribution $\left(\tau_{T 0} \gg \tau_{T 1}\right)$. The electron then travels from $A$ to trigger the release of the G-protein $(G)$ over a time $\tau_{R}$. Note that the terms elastic and inelastic refer only to energy exchange with the odorant.

IETS there is a strong contribution to this coupling from the Coulomb interaction between partial charges associated with oscillating atoms and a mobile electron[27, 28]. This same mechanism allows us to account for observed features of olfaction including the detection of oscillators buried inside a molecule (e.g. 2,6 di-t-butyl phenol [2]), and is compatible with 
our swipe card model: the long-ranged interaction can couple the mobile electron to the oscillator even with a loose fit.

The times characterising elastic $\left(\tau_{T 0}\right)$ and inelastic $\left(\tau_{T 1}\right)$ ET from $D$ to $A$ are central to the success or failure of Turin's mechanism. We treat $D$ and $A$ as single molecular orbitals with energies $\varepsilon_{D}$ and $\varepsilon_{A}$, coupled to each other by a weak hopping integral $t$, but not coupled to other electronic states. Since the hopping between $D$ and $A$ is slow on electronic time scales, the remaining electronic couplings must be very weak to prevent electron leakage. However, $D$ and $A$ will be coupled to oscillators in the odorant, receptor protein and the wider environment. The ET rate from $D$ to $A$ can be computed from standard theory [23, 24, 25, 29, 30, 31] but with the odorant oscillator treated explicitly. We consider one odorant oscillator of frequency $\omega_{o}$ which couples with strength $\gamma_{D}\left(\gamma_{A}\right)$ to $D(A)$. The environment is treated as many oscillators with frequencies $\omega_{q}$ and coupling strengths $\gamma_{q D}$ and $\gamma_{q A}$. The complete system is described by the Hamiltonian $\hat{H}=\hat{H}_{D}+\hat{H}_{A}+\hat{v}$, where $\hat{H}_{X}=|X\rangle\langle X|\left(\varepsilon_{X}+\hat{H}_{o s c}+\hat{H}_{e-o s c, X}\right)(X$ is $D$ or $A), \hat{v}=t(|D\rangle\langle A|+| A\rangle\langle D|)$ and $|D\rangle(|A\rangle)$ is an electronic state on $D(A)$. $\hat{H}_{o s c}=\left(\hat{a}^{\dagger} \hat{a}+\frac{1}{2}\right) \hbar \omega_{o}+\sum_{q}\left(\hat{a}_{q}^{\dagger} \hat{a}_{q}+\frac{1}{2}\right) \hbar \omega_{q}$ is the oscillator Hamiltonian for the odorant and environment, and $\hat{H}_{e-o s c, X}=\gamma_{X}\left(\hat{a}+\hat{a}^{\dagger}\right)+\sum_{q} \gamma_{q X}\left(\hat{a}_{q}+\hat{a}_{q}^{\dagger}\right)$ couples the electron to the oscillators. The eigenstates of $\hat{H}_{o s c}$ are $|n N\rangle$, where $n$ is the odorant oscillator occupancy and $N$ corresponds to a set of environment oscillator occupancies $\left\{n_{q}\right\}$. The eigenstates of $\hat{H}_{X}$ are $\left|\Psi_{X n N}\right\rangle=\exp \left(u_{X}\left(\hat{a}-\hat{a}^{\dagger}\right)+\sum_{q} u_{q X}\left(\hat{a}_{q}-\hat{a}_{q}^{\dagger}\right)\right)|X n N\rangle$ and have eigenvalues $E_{X n N}=\varepsilon_{X}+\left(n+\frac{1}{2}-u_{X}^{2}\right) \hbar \omega_{o}+\sum_{q}\left(n_{q}+\frac{1}{2}-u_{q X}^{2}\right) \hbar \omega_{q}$. The states $|X n N\rangle$ are products of unperturbed electronic and oscillator basis states, $u_{X}=\gamma_{X} / \hbar \omega_{o}$ and $u_{q X}=\gamma_{q X} / \hbar \omega_{q}$. The times $\tau_{T 0}$ and $\tau_{T 1}$ follow from the standard golden rule result for the coupling of the system with the electron on $D$ and odorant oscillator in its ground state to that with the electron on $A$ and odorant oscillator in excited state $|n\rangle: 1 / \tau_{T n}=(2 \pi / \hbar) \sum_{N N^{\prime}} P_{N}\left|\left\langle\Psi_{D 0 N}|\hat{v}| \Psi_{A n N^{\prime}}\right\rangle\right|^{2}$ where $P_{N}$ is the probability that the system starts in state $\left|\Psi_{D 0 N}\right\rangle$. After making standard approximations for an electron coupled to a bath of phonons [31, 32], and taking the background fluctuations to be of low frequency, we get the Marcus-type expression

$$
\frac{1}{\tau_{T n}}=\frac{2 \pi}{\hbar} t^{2} \frac{\sigma_{n}}{\sqrt{4 \pi k_{B} T \lambda}} \exp \left(-\frac{\left(\epsilon_{n}-\lambda\right)^{2}}{4 k_{B} T \lambda}\right)
$$

where $\sigma_{n}=\exp (-S) S^{n} / n$ !, $S=\left(u_{D}-u_{A}\right)^{2}$ (a Huang-Rhys factor), $\epsilon_{n}=\varepsilon_{D}-\varepsilon_{A}-n \hbar \omega_{o}$, $\beta=1 / k_{B} T, \lambda=\sum_{q} S_{q} \hbar \omega_{q}$ (reorganisation energy), and $S_{q}=\left(u_{q D}-u_{q A}\right)^{2}$. 


\begin{tabular}{c|c|c|c|c} 
Quantity & $\hbar \omega_{o}$ & $S$ & $\lambda$ & $|t|$ \\
\hline Value & $200 \mathrm{meV}$ & 0.01 & $30 \mathrm{meV}$ & $1 \mathrm{meV}$
\end{tabular}

TABLE I: Estimated values for the physical quantities needed to compute $\tau_{T 0}$ and $\tau_{T 1}$. See text for explanation of their values.

We now estimate values for the parameters (Table II). The interesting range for $\hbar \omega_{o}$ in olfaction is about $70 \mathrm{meV}$ to $400 \mathrm{meV}[18]$, so a typical value is $200 \mathrm{meV}$. To estimate the Huang-Rhys factor $S$ we introduce a physical mechanism for the electron-oscillator interaction based on the long-ranged electrostatic interaction between the electron and odorant atomic partial charges. The definition $S=\left(u_{D}-u_{A}\right)^{2}$ is equivalent to $S=\Delta F^{2} / 2 \hbar M_{o} \omega_{o}^{3}$ where $\Delta F$ is the change in force on the odorant oscillator as a result of the electronic transition [33, 34]. We treat the oscillator as a dipole with charges $\pm q e$ and compute the forces on the oscillator when the electron is on $A$ and $D$ (treated as pointlike), giving

$$
S=4 q^{2} \frac{m_{e}}{M_{o}}\left(\frac{R y}{\hbar \omega_{o}}\right)^{3}\left(\frac{\hat{R}_{D} \cdot \hat{p}}{\left(R_{D} / a_{0}\right)^{2}}-\frac{\hat{R}_{A} \cdot \hat{p}}{\left(R_{A} / a_{0}\right)^{2}}\right)^{2}
$$

where $\hat{p}$ is the direction of the dipole, $\vec{R}_{D}$ is the vector from $D$ to the dipole, $\vec{R}_{A}$ is the vector from $A$ to the dipole, $m_{e}$ is the electron mass, $R y$ the Rydberg and $a_{0}$ the Bohr radius. Setting $q=0.2$ (a typical partial atomic charge in a polar molecule), $m_{e} / M_{o}=1 / 15000$ (using a representative atomic mass for light elements), $\hbar \omega_{o}=200 \mathrm{meV}, \hat{R}_{D} \cdot \hat{p}=-\hat{R}_{A} \cdot \hat{p}=1$ and $R_{D}=R_{A}=6 a_{0}$ gives $S \sim 0.01$.

We assume the odorant $(M)$ contacts $D$ and $A$ but interacts with them only weakly with hopping integral $v$. By considering the resulting admixtures of an $M$ state with energy $\varepsilon_{M}$ with those of $D$ and $A$ we obtain an effective hopping integral between $D$ and $A$ ( $\left.t=v^{2} /\left(\varepsilon_{M}-\varepsilon_{A}\right)\right)$. If $\varepsilon_{M}$ corresponds to a LUMO while $\varepsilon_{D}$ and $\varepsilon_{A}$ correspond to HOMOs then the difference $\varepsilon_{M}-\varepsilon_{A}$ can be as large as $10 \mathrm{eV}$. The hopping integrals can be estimated for known molecular structures. Whilst the odorant structure is known, the donor and acceptor structures interacting with it are unknown, and we have to make an educated guess. If the bonds between $M$, and $D$ and $A$ are no stronger than hydrogen bonds, we can put a rough upper bound on the associated hopping integrals of order $0.1 \mathrm{eV}$, and hence obtain $t \sim 1 \mathrm{meV}$. Our final conclusions are not sensitive to this value.

Reorganisation energies are typically of order $1 \mathrm{eV}$, especially in hydrated systems, which 
would result in the elastic channel being much faster than the inelastic. But much smaller values have been found, and olfactory receptors are hydrophobic. Experiments on charge separation in mutant reaction centers of the photosynthetic bacteria Rhodobacter capsulatus show reorganisation energies at room temperature below $0.03 \mathrm{eV}[35]$. A generally low value for odorant receptors would be a result of evolutionary optimization leading to almost no reorganization during the transition. This requires that $D$ and $A$ are not too close to the aqueous medium in the cell to prevent significant coupling to the polarization of the water; thus we conjecture that $D$ and $A$ must lie well within the lipid bilayer region (see Fig. (1). The reorganisation energy can also be reduced if electronic states on $D$ and $A$ are extended in space [29], so residues with delocalized electrons may be candidates. (For example, the conserved[36] tryptophan on helix 4 and 3 phenylalanines on helix 3 . The surrounding highly variable residues could modify their redox potentials, producing different receptors. ) We take a value for the reorganisation energy of $0.03 \mathrm{eV}$ for the table of values.

Substituting the values in Table 1 into Eq. 1 for the case of resonance $\left(\varepsilon_{D}-\varepsilon_{A}=\hbar \omega_{o}\right)$ we get $\tau_{T 0} \sim 87 \mathrm{~ns}$ and $\tau_{T 1} \sim 1.3 \mathrm{~ns}$, which satisfies the condition $\tau_{T 1} \ll \tau_{T 0}$, and shows that the overall time for odor recognition is not limited by the discrimination process. Increasing the reorganization energy to just $50 \mathrm{meV}$ would make $\tau_{T 1}>\tau_{T 0}$. Thus, provided the reorganisation energy can be made not much bigger than $k_{B} T$, we can obtain a large signal to noise ratio. We note that the theory remains unaltered if a proton tunnel from $D$ to $A$, but the parameters $t, \varepsilon_{D}$ and $\varepsilon_{A}$ will be modified.

Our analysis indicates that Turin's model is physically viable provided the receptor has certain properties (notably, very low reorganization energy) within ranges known from other biomolecular systems. Our model shows that the overall charge transfer rate is sufficient to permit detection on the observed timescales, and the inelastic signal can be made sufficiently large relative to the elastic signal for there to be an acceptable signal to noise ratio. Lack of information on local receptor structure limits what can be verified. Our model illustrates a more general idea of how molecules can actuate receptors selectively. Lock and key models rely on docking for discrimination, and mechanical mechanisms for actuation. Selective docking does have a role in our class of swipe card models, but the crucial discrimination and non-mechanical actuation processes are different. 


\section{Acknowledgments}

Many entertaining and instructive conversations with Luca Turin and helpful comments from Rudolph Marcus are gratefully acknowledged. JB and AH are supported by the EPSRC through the IRC in Nanotechnology.

[1] J. C. Leffingwell, Leffingwell Reports 5, 1 (2001).

[2] D. Rowe, ed., Chemistry and Technology of Flavours and Fragrances (Blackwells, 2005), chap. 11.

[3] R. Axel, Angew. Chem. Int. Ed. 44, 6111 (2005).

[4] L. Buck, Angew. Chem. Int. Ed. 44, 6128 (2005).

[5] W. Bialek, Ann. Rev. Biophys. Biophys. Chem. 16, 455 (1987).

[6] R. B. Silverman, The Organic Chemistry of Enzyme-Catalyzed Reactions (Elsevier, 2002).

[7] D. Wallace, A. M. Stoneham, A. Testa, A. H. Harker, and M. M. D. Ramos, Molecular Simulation 2, 385 (1993).

[8] D. Wrobel, U. Wannagat, and U. Harder, Chemical Monthly 113, 381 (1982).

[9] G. M. Dyson, Chem. Ind. 57, 647 (1938).

[10] R. H. Wright, The sense of smell (CRC press, Boca Raton, Florida, USA, 1982).

[11] J. Lambe and R. C. Jaklevic, Phys. Rev. 165, 821 (1968).

[12] C. J. Adkins and W. A. Phillips, J. Phys. C: Solid State Phys. 18, 1313 (1985).

[13] L. Turin, Chem. Senses 21, 773 (1996).

[14] L. J. W. Haffenden, V. A. Yaylayan, and J. Fortin, Food Chemistry 73, 67 (2001).

[15] A. Keller and L. B. Vosshall, Nature Neuroscience 7, 337 (2004).

[16] B. R. Havens and C. E. Meloan, Food Flavors: Generation, Analysis and Process Influence 37, 497 (1995).

[17] E. Brenna, C. Fuganti, and S. Serra, Tetrahedron: Asymmetry 14, 1 (2003).

[18] L. Turin, J. Theor. Biol. 216, 367 (2002).

[19] S.-y. Takane and J. B. O. Mitchella, Org. Biomol. Chem. 2, 3250 (2004).

[20] M. J. Frisch, G. W. Trucks, H. B. Schlegel, G. E. Scuseria, M. A. Robb, J. R. Cheeseman, J. A. Montgomery, Jr., T. Vreven, K. N. Kudin, J. C. Burant, et al., Gaussian 03, Revision 
C.02, Gaussian, Inc., Wallingford, CT, 2004.

[21] D. R. Lide, ed., CRC Handbook of Chemistry and Physics (CRC Press, 2005), 86th ed.

[22] P. Atkins and J. de Paula, Atkins' Physical Chemistry (OUP, 2002).

[23] R. A. Marcus, Annu. Rev. Phys. Chem. 15, 155 (1964).

[24] J. Ulstrup, Charge Transfer Processes in Condensed Media, vol. 10 of Lecture Notes in Chemistry (Springer-Verlag, 1979).

[25] D. S. Bendall, ed., ProteinElectronTransfer (Bios Scientific Publishers, 1996).

[26] H. B. Gray and J. R. Winkle, Annu Rev. Biochem. 65, 537 (1996).

[27] J. R. Kirtley, D. J. Scalapino, and P. K. Hansma, Phys. Rev. B 14, 3177 (1976).

[28] J. R. Kirtley and P. Soven, Phys. Rev. B 19, 1812 (1979).

[29] R. A. Marcus, J. Chem. Phys. 24, 966 (1956).

[30] R. A. Marcus, J. Chem. Phys. 43, 679 (1965).

[31] X. Song and R. A. Marcus, J. Chem. Phys. 99, 7768 (1993).

[32] C. P. Flynn and A. M. Stoneham, Phys. Rev. B 1, 3966 (1970).

[33] K. Huang and A. Rhys, Proc. Royal Soc. A204, 406 (1950).

[34] A. M. Stoneham, Theory of Defects in Solids (Oxford University Press, 2001).

[35] Y. Jia, T. J. DiMagno, C.-K. Chan, Z. Wang, M. Du, D. K. Hanson, M. Schiffer, J. R. Norris, G. R. Fleming, and M. S. Popov, J. Phys. Chem. 97, 13180 (1993).

[36] T. Fuchs, G. Glusman, S. Horn-Saban, D. Lancet, and Y. Pilpel, Hum. Genet. 108, 1 (2001). 